\title{
Funcionalidad familiar, autovalencia y bienestar psicosocial de adultos mayores
}

\author{
Claudia Troncoso Pantoja ${ }^{1, a}$, Nayadeth Soto-López ${ }^{1}$
}

\section{RESUMEN}

Objetivo: Relacionar la funcionalidad familiar con la autovalencia y el bienestar psicosocial de personas mayores pertenecientes a la comuna de Huechuraba, Santiago, Chile, durante el año 2016.

Materiales y métodos: Estudio descriptivo, de corte transversal, correlacional. El muestreo fue intencionado, completando tamaño muestral en 60 adultos mayores voluntarios que cumplieron los criterios de selección. Para la recolección de información se utilizaron el Apgar Familiar, Escala de Whoqol-Bref y EFAM (Examen de Funcionalidad del Adulto Mayor). Para el análisis de resultados se utilizó estadígrafos descriptivos y pruebas de asociación Chi-cuadrado y la prueba de U de MannWhitney.

Resultados: Más del 70 \% de los participantes presentaron familias muy funcionales y sólo el 3 \% pertenecieron a familias con graves disfunciones. 45 \% fueron autovalentes con riesgo y $16.7 \%$ presentaron riesgo de dependencia. No se encontró relación entre funcionalidad familiar y salud física; sí se presenta asociación entre funcionalidad familiar, salud psicológica y relaciones interpersonales.

Conclusiones: Existe relación entre funcionalidad familiar y nivel de autovalencia, salud psicológica, relaciones interpersonales y entorno, debido a que aquellos que pertenecen a familias con una mejor dinámica interna, disfrutan de una mejor calidad de vida.

Palabras claves: Adulto mayor; relación familiar; dependencia; Psicología Social (Fuente: DeCS BIREME).

\section{Family functionality, autonomy and psychosocial welfare in the elderly}

\section{ABSTRACT}

Objective: To relate family functionality to autonomy and psychosocial welfare of elderly people living in the Huechuraba commune in Santiago, Chile, during the year 2016.

Materials and methods: A descriptive, cross-sectional, correlational study with a purposive sample of 60 elderly volunteers who fulfilled the screening criteria. For data collection, the Family APGAR test, WHOQOL-BREF scale and Functional Assessment of the Elderly (EFAM) were used. For the analysis of the results, descriptive statistics and association tests such as chi-square and Mann-Whitney $U$ test were used.

Results: Over $70 \%$ of the participants had very functional families and only $3 \%$ belonged to severely dysfunctional families. Forty-five percent ( $45 \%$ ) of the participants were autonomous but at risk of being dependent and $16.7 \%$ were at risk of being dependent. No relationship between family functionality and physical health was observed; however, there is an association between family functionality, psychological health and interpersonal relationships.

Conclusions: There is a relationship between family functionality and level of autonomy, psychological health, interpersonal relationships and environment, because elderly people who belong to families with better internal dynamics enjoy a better quality of life.

Keywords: Aged; family relations; dependency; psychology, social (Source: MeSH NLM).

1. Universidad Católica de la Santísima Concepción, Facultad de Medicina, Programa de Magister en Promoción de Salud Familiar y Comunitaria. Concepción, Chile.

a. Investigador asociado, Centro de Investigación en Educación y Desarrollo (CIEDE-UCSC). Concepción, Chile. 


\section{INTRODUCCIÓN}

La Organización Mundial de Salud declara el rápido envejecimiento de la población en estos últimos tiempos, proyectando para el año 2050, un incremento poblacional que considerará hasta 2000 millones de personas mayores de 60 años $^{(1)}$. Es por esto que la misma institución, a través de lo que ha definido como "envejecimiento activo", tiende a promocionar el fortalecimiento de políticas públicas dirigidas a este grupo etario, que incluya entre otros temas, la formación de capital humano avanzado ${ }^{(2)}$, presentando como fin último el mejorar la calidad de vida de estas personas.

Si bien definir calidad de vida puede ser un concepto muy heterogéneo, desde el paradigma médico, se vincula al hecho de presentar una adecuada salud mental o ausencia de enfermedad, así como un estado de felicidad pleno, "buena vida", independencia funcional, entre otras definiciones ${ }^{(3)}$.

Es por esto que mejorar la calidad de vida de las personas, en especial de los adultos mayores, es el objetivo transversal que presentan los equipos de salud en las diversas actividades realizadas, debido a que, a mayor y mejor calidad de vida, menor presencia de complicaciones, como puede ser una discapacidad física ${ }^{(4)}$. Está condicionada por diversos factores como el estado de salud, las relaciones interpersonales, número de hijos, escolaridad o la edad. Esto se explica debido a que, a mayor cantidad de años vividos, se presenta una disminución de la apreciación de la calidad de vida ${ }^{(5-8)}$.

Es por esto que la calidad de vida de una persona mayor ofrece una directa concordancia a la funcionalidad de esta última. Es individual y tiende a declinar a medida que transcurren los años, iniciándose generalmente en forma más acentuada a partir de los 65 años ${ }^{(9,10)}$.

Clásicamente se ha definido a la independencia funcional o funcionalidad como la capacidad de realizar las actividades diarias, permitiéndoles a los adultos mayores el vivir y subsistir en forma independiente ${ }^{(11)}$. Estas habilidades se relacionan a la frecuencia de actividad física y de integración social, lo que favorece su calidad de vida y limita la aparición de modificaciones psicosociales ${ }^{(12)}$. Sin embargo, su sensación de bienestar y autovalencia no solo se limita a algunos aspectos físicos. La familia, en especial, la estabilidad que representa, responde a la estructura, ordenamiento y comunicación típica entre sus integrantes, transmitidos entre generaciones ${ }^{(13)}$. Se identifica como el establecimiento de mayor importancia para este grupo etario, cumpliendo una representación de conservación y garantía de un buen estado de salud ${ }^{(11)}$. Es por esto que se describe que personas mayores más limitadas en su acompañamiento social, especialmente de familia directa, presentan un mayor deterioro en su disposición frente a la vida y, por lo tanto, afecta su bienestar ${ }^{(14,15)}$.

En la actualidad, este efecto de bienestar en personas mayores puede ser medido a través de instrumentos de recolección de información, como el Apgar Familiar, instrumento que evalúa como en la familia se percibe el nivel de funcionamiento o las relaciones interpersonales entre sus miembros ${ }^{(16)}$; la escala de Whoqol-Bref, instrumento genérico que permite medir calidad de vida en este grupo etario ${ }^{(17)} \mathrm{y}$ el examen de funcionalidad del adulto mayor (EFAM) usado en Chile en personas mayores de 65 años para identificar si puede valerse por sí mismo para actividades instrumentales de la vida diaria ${ }^{(18)}$.

Esta investigación posibilita seguir fortaleciendo la generación de conocimiento en este grupo etario, entrelazando la concepción de calidad de vida con la funcionalidad de estas personas y la estrecha relación que se produce con la familia, que permite mejorar la sensación de bienestar y autovalencia en estas personas, entregando un aporte a la salud familiar. Es por esto que el objetivo es describir la relación existente entre la funcionalidad familiar con la autovalencia y el bienestar psicosocial de personas mayores pertenecientes a la comuna de Huechuraba, Santiago, Chile, durante el año 2016.

\section{MATERIALES Y MÉTODOS}

Las metas de este estudio se cumplen utilizando el diseño descriptivo, de corte transversal, correlacional para profundizar en las variables funcionalidad familiar, autovalencia y bienestar psicosocial. La población diana correspondió a un grupo de personas mayores, asistentes a grupos establecidos para su grupo etario de la comuna de Huechuraba (del mapudungún: "Lugar donde nace la greda"), ubicado en el área norte de Santiago, Chile, con una superficie total de 4430 hectáreas de las cuales 3180 corresponde a zona urbana. Su densidad poblacional es de 17,7 habitantes por hectárea ${ }^{(19)}$.

Para ingresar al estudio, los participantes debían presentar como edad 65 años o más, asistir a grupos de adultos mayores establecidos en la comuna, capacidad cognitiva y funcional para contestar preguntas de los instrumentos de recolección de datos: Apgar Familiar, escala de WhoqolBref y EFAM. A lo anterior, firmar el consentimiento informado propio de este estudio.

El muestreo fue no probabilístico de sujetos voluntarios. La muestra quedó constituida por 60 personas mayores: $85 \%$ mujeres y $15 \%$ varones, a quienes se les aplicó instrumentos antes mencionados, previa prueba piloto a cinco adultos mayores.

El análisis de los datos se realizó utilizando el Programa SPSS 12, utilizando estadígrafos descriptivos como medias y desviaciones estándar. Para las pruebas de asociaciones se usó Chi-cuadrado y U de Mann-Whitney, ambas con un nivel de significancia de $5 \%$.

Los aspectos éticos fueron regulados a través de la aprobación de la investigación por parte del Comité de Ética de la Universidad Católica de la Santísima Concepción, Chile. El consentimiento informado aplicado identificaba el objetivo del estudio, el tipo de participación en este, su opción a retirarse sin coerción, la confiabilidad y respaldo de sus datos personales, además del anonimato. 


\section{RESULTADOS}

La tabla 1 entrega los antecedentes demográficos de los participantes del estudio. Este se concentra en mujeres, mayores de 70 años, principalmente viudas o casadas. En conjunto no se identifican como maltratados; presentan familias biparentales con un promedio de tres hijos y presencia de patologías cardiovasculares. El $85 \%$ no fuma y $75 \%$ no consume alcohol.

Tabla 1. Antecedentes demográficos de población estudiada. Huechuraba, 2016

\begin{tabular}{|c|c|}
\hline Muestra (n): & 60 \\
\hline \multicolumn{2}{|l|}{ Sexo } \\
\hline Masculino & $9(15 \%)$ \\
\hline Femenino & $51(85 \%)$ \\
\hline Edad & $71+/-4$ \\
\hline \multicolumn{2}{|l|}{ Estado civil } \\
\hline Casado & $24(40 \%)$ \\
\hline Viudo & $24(40 \%)$ \\
\hline Soltero & $6(10 \%)$ \\
\hline Separado & $5(8,3 \%)$ \\
\hline Conviviente & $1(1,7 \%)$ \\
\hline \multicolumn{2}{|l|}{ Hábito tabáquico } \\
\hline Sí & $9(15 \%)$ \\
\hline No & $51(85 \%)$ \\
\hline \multicolumn{2}{|l|}{ Consumo de alcohol } \\
\hline Sí & $15(25 \%)$ \\
\hline No & $45(75 \%)$ \\
\hline \multicolumn{2}{|l|}{ Percepción de maltrato } \\
\hline Sí & $3(5 \%)$ \\
\hline No & $57(95 \%)$ \\
\hline \multicolumn{2}{|l|}{ Tipo de familia } \\
\hline Biparental & $22(36,7 \%)$ \\
\hline Monoparental & $11(18,3 \%)$ \\
\hline Unipersonal & $8(13,3 \%)$ \\
\hline Biparental extendida & $4(6,7 \%)$ \\
\hline Monoparental extendida & $15(25 \%)$ \\
\hline Número de hijos & $3,3+/-1,5$ \\
\hline \multicolumn{2}{|l|}{ Presencia de patología } \\
\hline Enfermedad cardiovascular & $54(90 \%)$ \\
\hline $\begin{array}{l}\text { Enfermedad crónica no } \\
\text { cardiovascular }\end{array}$ & $3(5 \%)$ \\
\hline Sin patología declarada & $3(5 \%)$ \\
\hline
\end{tabular}


La tabla 2 identifica la funcionalidad y autovalencia de los adultos mayores. Se presenta significancia entre la funcionalidad familiar y la autovalencia, identificando que las personas mayores participantes, integrantes de familias muy funcionales, mostraron riesgo reducido $(6.5 \%)$ en su autovalencia.

Tabla 2. Funcionalidad familiar y autovalencia de los adultos mayores. Huechuraba, 2016

\begin{tabular}{|c|c|c|c|c|c|}
\hline \multirow{4}{*}{$\begin{array}{l}\text { Funcionalidad } \\
\text { familiar }\end{array}$} & & \multicolumn{3}{|c|}{ Autovalencia } & \multirow{2}{*}{$\begin{array}{c}\text { Total } \\
\text { personas } \\
\text { mayores }\end{array}$} \\
\hline & & $\begin{array}{c}\text { Autovalente } \\
\text { sin riesgo }\end{array}$ & Autovalente con riesgo & $\begin{array}{l}\text { Persona en } \\
\text { riesgo de } \\
\text { dependencia }\end{array}$ & \\
\hline & Muy funcional & $20(43,5 \%)$ & $23(50,0 \%)$ & $3(6,5 \%)$ & 46 \\
\hline & $\begin{array}{c}\text { Moderadamente } \\
\text { funcional / grave } \\
\text { disfunción }\end{array}$ & $3(21,4 \%)$ & $4(28,6 \%)$ & $7(50,0 \%)$ & 14 \\
\hline Total & & $23(38,3 \%)$ & $27(45,0 \%)$ & $10(16,7 \%)$ & 60 \\
\hline
\end{tabular}

La tabla 3 establece la capacidad funcional familiar y la salud física de los adultos mayores. Para esta medición, no se encontró relación entre la funcionalidad familiar y la salud física, aunque se identifica que las familias más funcionales presentan una mejor salud física.

Tabla 3. Funcionalidad familiar y salud física de los adultos mayores. Huechuraba, 2016

$\begin{array}{ccccc} & \text { Mínimo } & \text { Máximo } & \text { Mediana } & \text { Media +/- DE } \\ \text { Familia muy funcional } & 31 & 94 & 63 & 60,7+/-4 \\ \begin{array}{c}\text { Familia moderadamente funcional } \\ \text { / grave disfunción }\end{array} & 25 & 81 & 56 & 54,9+/-8,5\end{array}$

Fuente: Elaboración propia

p-valor: 0,161

En la tabla 4 se presenta la funcionalidad familiar y salud psicológica de los participantes. Se identificó una correlación entre estas variables, situación que se concentra en familias con una mayor funcionalidad.

Tabla 4. Funcionalidad familiar y salud psicológica de los adultos mayores. Huechuraba, 2016

$\begin{array}{ccccc} & \text { Mínimo } & \text { Máximo } & \text { Mediana } & \text { Media +/- DE } \\ \begin{array}{c}\text { Familia muy funcional } \\ \text { Familia moderadamente funcional } \\ \text { / grave disfunción }\end{array} & 25 & 94 & 69 & 67,9+/-4 \\ & 25 & 81 & 59,5 & 57,2+/-9,8\end{array}$

Fuente: Elaboración propia

p-valor: 0,032

Finalmente, la tabla 5 establece las relaciones interpersonales y el funcionamiento familiar de los sujetos estudiados. Se presenta una correspondencia, ya que las familias muy funcionales tienden a presentar mejores vínculos entre personas que los individuos provenientes de familias menos funcionales.

Tabla 5. Funcionalidad familiar y relaciones interpersonales de los adultos mayores. Huechuraba, 2016

$\begin{array}{ccccc} & \text { Mínimo } & \text { Máximo } & \text { Mediana } & \text { Media +/- DE } \\ \text { Familia muy funcional } & 19 & 81 & 50 & 46+/-5 \\ \begin{array}{c}\text { Familia moderadamente funcional } \\ \text { / grave disfunción }\end{array} & 6 & 69 & 31 & 29,8+/-11,5\end{array}$

Fuente: Elaboración propia

p-valor: 0,012 


\section{DISCUSIÓN}

El estudio se focalizó en la necesidad de identificar la capacidad funcional de un grupo de personas mayores y como diversos factores condicionan esta funcionalidad.

Al entrelazar los conceptos de autovalencia o autonomía, el estudio de Massone et al ${ }^{(20)}$ profundiza en la importancia que para personas mayores les resulta el ser independientes, especialmente con su grupo familiar. Esta situación se replica en personas semivalente ${ }^{(21)}$. En nuestro estudio, las familias muy funcionales presentaban autovalencia, aunque una mayor proporción de autonomía moderada.

En cuanto a la salud física, el estudio de González et al, plantea que la condición de salud que presenta el adulto mayor y los factores de riesgo que condicionan el envejecimiento, es sólo un umbral, que permite identificar los tipos de envejecimiento ${ }^{(22)}$. En esta investigación, no se encontró un nivel de significancia entre la funcionalidad familiar y la salud física de las personas participantes, sin embargo, hay una leve tendencia a que familias más funcionales responden a una mejor salud física y por lo mismo, una mejor integración social. Esto último coincide con el estudio de Landinez et al ${ }^{(23)}$, el cual plantea que un envejecimiento activo permite a las personas potenciar su bienestar físico, social y mental, participando socialmente de acuerdo a sus necesidades, deseos y capacidades, mejorando su calidad de vida.

El estudio de Vivaldi et al (24) establece que el bienestar psicológico de la muestra estudiada, presenta una mayor relación con el apoyo social. Nuestros resultados coinciden con esto último, además de direccionar hacia la significancia que se presenta entre familias funcionales y su salud psicológica. Arias en su artículo El apoyo social en la vejez: la familia, los amigos y la comunidad ${ }^{(25)}$ profundiza en el soporte social de personas mayores, incluyendo a las familias de estos, concluyendo que el apoyo social promueve el desarrollo de potencialidades de las personas mayores.

Entendiendo que los lazos sociales favorecen el intercambio afectivo positivo, el mayor contacto y fortalecimiento estructural y funcional de relaciones interpersonales, permiten vigorizar este apoyo ${ }^{(26,27)}$. En nuestra investigación y en concordancia con el trabajo realizado por Alfonso et $\mathrm{al}^{(28)}$, las personas mayores provenientes de familias más eficaces, presentan mejores relaciones interpersonales, lo que fortalece finalmente su calidad de vida.

El estudio presenta la peculiaridad de estar desarrollado en un grupo de adultos mayores de una zona urbana que se encontraban participando en centro de reunión formal para actividades propias de su grupo etario. Esta situación se debe resaltar, ya que la investigación liderada por Durán et al ${ }^{(12)}$, refieren que la integración social, favorece las habilidades funcionales de estas personas, situación que coincide con nuestros resultados.

Un aspecto que no se sopesó en este estudio y que podría tomarse en consideración en próximas investigaciones, es el rango de edad de las personas mayores, ya que en la actualidad y con las expectativas de vida, una persona de 70 , 80 o 90 años, identifica de distintas maneras condicionantes en su calidad de vida, situación que lo que es reconocido por algunos autores ${ }^{(29,30)}$.

En conclusión, la calidad de vida de personas mayores está supeditada por la funcionalidad familiar, identificando que la autovalencia y el bienestar psicosocial de estas personas se presenta de mayor y mejor forma en familias funcionales.

Agradecimiento: A todas las personas mayores de la comuna de Huechuraba, Chile, que participaron en esta investigación.

\section{REFERENCIAS BIBLIOGRÁFICAS}

1. Organización Mundial de la Salud. Envejecimiento y ciclo de vida. [Web]. [Acceso en agosto de 2017]. Disponible en: http: / / www. who.int/features/factfiles/ageing/ageing_ facts/es/

2. Marín P. Reflexiones para considerar en una política pública de salud para las personas mayores. Rev med. Chile. 2007; 135(3): 392-398. Disponible en: http://dx.doi.org/10.4067/ S0034-98872007000300016

3. Estrada A, Cardona D, Segura A, Chavarriaga L, Ordoñez $\mathrm{J}$, Osorio J. Calidad de vida de los adultos mayores de Medellín. Biomédica. 2011; 31(4): 492-502. Disponible en: http:// www.scielo.org.co/scielo. php?pid=S012041572011000400004\&script=sci_abstract\&tlng=es

4. Botero B, Pico M. Calidad de vida relacionada con la salud (CVRS) en adultos mayores de 60 años: una aproximación teórica. Hacia la Promoción de la Salud. 2007; 12(1): 11-24. http: / / www.scielo.org.co/scielo.php?pid=S0121$75772007000100002 \&$ script=sci_abstract\&tlng=es

5. Peña P, Terán T, Moreno A, Bazán C. Autopercepción de la calidad de vida del adulto mayor en la Clínica de Medicina Familiar Oriente del ISSSTE. Rev Esp Med Quir. 2009; 14(2): 53-61. Disponible en:http://www.redalyc.org/articulo. oa?id=47312308002

6. Dueñas D, Bayarre H, Triana E, Rodríguez V. Calidad de vida percibida en adultos mayores de la provincia de matanzas. Rev Cubana Med Gen Integr. 2009; 25(2): 0-0. Disponible en: http://scielo.sld.cu/scielo.php?script=sci_ arttext\&pid=S0864-21252009000200002

7. Dueñas D, Bayarre H, Triana E, Rodríguez V. Percepción de salud en adultos mayores de la provincia Matanza. Rev Cubana Med Gen Integr. 2011; 27(1): 10-22. Disponible en: http://scielo.sld.cu/scielo.php?script=sci_ arttext\&pid=S0864-21252011000100002

8. De la Fuente $T$, Quevedo $E$, Jiménez A, Zavala M. Funcionalidad para actividades de la vida diaria en el adulto mayor de zonas rurales. Archivos de Medicina Familiar. 2010; 12(1): 1-4. Disponible en:http://www.redalyc.org/articulo. oa?id $=50719083001$

9. Álvarez $\mathrm{K}$, Delgado $\mathrm{A}$, Naranjo J, Pérez $M$, Valdés $\mathrm{A}$. Evaluación funcional del adulto mayor en la comunidad. Rev Ciencias Médicas. 2012; 16(2): 124-137. Disponible en: http://scielo.sld.cu/scielo.php?script=sci_ arttext\&pid $=\$ 1561-31942012000200010$

10. Placeres J, De León L, Delgado I. La familia y el adulto mayor. Rev. Med. Electrón. 2011; 33(4): 472-483. Disponible en: http://scielo.sld.cu/scielo.php?script=sci arttext\&pid=S1684-18242011000400010

11. Sanhueza M, Castro M, Merino J. Adultos mayores funcionales: un nuevo concepto en salud. Ciencia y Enfermería. 2005; 11(2): 17-21. Disponible en: http://dx.doi.org/10.4067/ S0717-95532005000200004 
12. Duran D, Valderrama J, Uribe A, Uribe J. Integración social y habilidades funcionales en adultos mayores. 2008; 7(1): 263-270. Disponible en: http://sparta.javeriana.edu. co/psicologia/publicaciones/actualizarrevista/archivos/ V07N0118.PDF

13. Zavala M, Ríos M, García G, Rodríguez C. Funcionalidad familiar y ansiedad en pacientes adultos con enfermedad crónica. AQUICHAN. 2009; 9(3): 257-270. Disponible en: http: / / www.redalyc.org/articulo.oa?id=74112147006

14. Cid M, Montes R, Hernández $O$. La familia en el cuidado de la salud. Rev. Med. Electrón. 2014; 36(4): 462-472. Disponible en: http://scielo.sld.cu/scielo.php?script=sci_ arttext\&pid=S1684-18242014000400008

15. Martínez O, Camarero O, González C, Martínez L. Calidad de vida del adulto mayor en un consultorio médico del municipio Jaruco. Revista de Ciencias Médicas de la Habana. 2016; 22(1): 30-41. Disponible en: http://revcmhabana.sld.cu/index.php/ rcmh/article/view/925/pdf_87

16. Suárez M, Alcalá M. Apgar Familiar: una herramienta para detectar disfunción familiar. Rev. Med. La Paz. 2014; 20(1): 53-57. Disponible en: http://www.scielo.org.bo/scielo. php?script=sci_arttext\&pid=S1726-89582014000100010

17. Kalfoss M, Law G, Molzahn A. The suitability of the WHOQOLBREF for canadian and norwegian older adults. A.E. Eur J Ageing. 2008; 5:77. Disponible en: https://www.ncbi.nlm. nih.gov/pubmed/28798564

18. Ministerio de Salud. Chile. [Web]. [Acceso en agosto 2017]. Disponible en: http://diprece.minsal.cl/le-informamos/ auge/examen-medicina-preventiva/

19. Municipalidad de Huechuraba. [Web]. [Acceso en marzo 2017]. Disponible en: https://www.huechuraba.cl/huechuraba/ historia.html

20. Massone F, Valdebenito X, Vogel N. Observaciones de la vejez en familia. Una aproximación desde la corresidencia intergeneracional. Gazeta de Antropología. 2010; 16(1): 1-10. Disponible en: http://www.ugr.es/ pwlac/G26_17Francisca_ Massone-Ximena_Valdebenito.html

21. Osorio P, Torrejon M, Meershon C, Anigstein M. Comprensión de la calidad de vida en personas mayores semivalentes en Chile. Salud \& Sociedad. 2011; 2(2): 203-217.

Disponible en: https://dialnet.unirioja.es/servlet/ articulo?codigo $=3899613$

22. González C, Ham-Chande R. Funcionalidad y salud: una tipología del envejecimiento en México. Salud pública de México. 2007; 49(4): 458. Disponible en: http://www.scielo.org. mx/scielo. php?script=sci_arttext\&pid=S0036-36342007001000003

23. Landinez N, Contreras K, Castro A. Proceso de envejecimiento, ejercicio y fisioterapia. Rev Cubana Salud Pública. 2012; 38(4): 562-580. Disponible en: http://www.scielosp.org/ scielo. php?pid=S0864-34662012000400008\&script $=$ sci_ abstract\&tlng=es

24. Vivaldi F, Barra E. Bienestar psicológico, apoyo social percibido y percepción de salud en adultos mayores. Ter Psicol. 2012;
30(2): 23-29. Disponible en: http://dx.doi.org/10.4067/ S0718-48082012000200002

25. Arias C. El apoyo social en la vejez: la familia, los amigos y la comunidad. Revista Kairós Gerontología. 2013; 16(4):313-329. Disponible en: https://revistas.pucsp.br/index.php/kairos/ article/view/20024

26. Fernández R, Manrique F. Rol de la enfermería en el apoyo social del adulto mayor. Enferme Global. 2010; 19: 1-9. Disponible en: http://revistas.um.es/eglobal/article/ view/107281

27. Aranda C, Pando M. Conceptualización del apoyo social y las redes de apoyo social. Revista IIPSI. 2013; 16(1): 233-245. Disponible en: http://revistasinvestigacion.unmsm.edu.pe/ index.php/psico/article/viewFile/3929/3153

28. Alfonso L, Soto D, Santos N. Calidad de vida y apoyo social percibido en adultos mayores. Rev. Ciencias Médicas de Pinar del Río 2016; 20(1): 0-0. Disponible en: http://scielo.sld.cu/ scielo.php?script=sci_arttext\&pid=S1561-31942016000100012

29. Pinillos Y, Prieto E, Herazo Y. Nivel de participación en actividades sociales y recreativas de personas mayores en Barranquilla, Colombia. Rev salud pública. 2013; 15(6): 825-833. Disponible en: http://www.redalyc.org/articulo. oa?id=42231751005

30. Torres M, Quezada M, Rioseco R, Ducci M. Calidad de vida de adultos mayores probres de viviendas básicas: Estudio comparativo mediante uso de WHOQoL-BREF. Rev Méd Chile. 2008; 136(3): 325-333. Disponible en http://dx.doi. org/10.4067/S0034-98872008000300007

Fuentes de Financiamiento:

Este artículo ha sido financiado por la autora.

\section{Conflicto de Intereses:}

La autora declara no tener ningún conflicto de interés.

\section{Correspondencia:}

Claudia Troncoso Pantoja

Dirección: Alonso de Ribera 2850. Concepción. Chile.

Teléfono: 56-041-2345427 - 56-041-2345416

Correo electrónico: ctroncosop@ucsc.cl

Recibido: 01 de setiembre de 2017 Evaluado: 03 de setiembre de 2017 Aprobado: 25 de setiembre de 2017

(c) La revista. Publicado por Universidad de San Martín de Porres, Perú. (c) вү Licencia de Creative Commons Artículo en acceso abierto bajo términos de Licencia Creative Commons Atribución 4.0 Internacional. (http://creativecommons.org/licenses/by/4.0/)

ORCID iDs

Claudia Troncoso Pantoja Nayadeth Soto López https: / / orcid.org/0000-0002-8433-5750

https://orcid.org/0000-0002-5902-3720 